\title{
A self-energy approach to the energy loss in STEM
}

\author{
A Rivacoba, P M Echenique and N Zabala \\ Departamento de Fisica de Materiales, Facultad de Química, Universidad del Pais Vasco- \\ Euskal Herriko Unibertsitatea, Apdo 1072, 20080 San Sebastian, Spain
}

\begin{abstract}
From a self-energy approach, a general expression for the energy loss probability in scanning transmission electron microscopy is derived. As an application, the coupling between a small particle and the supporting surface is studied.
\end{abstract}

In recent years scanning transmission electron microscopy (STEM) has proved to be useful in the study of the behaviour of microstructures. In particular, small particles $(10-100 \AA)$ have attracted increasing interest due to their applications in catalysis and microelectronics. This problem has been widely studied both experimentally and theoretically [1-10].

The probability $P(\omega)$ of losing energy $\omega$ is related to the energy loss rate $\gamma$ experienced by the particle. This quantity is given by the imaginary part of the particle self-energy $\Sigma_{0}$; $\gamma=-2 \operatorname{Im} \Sigma_{0}$ (we use atomic units).

In the pair approximation $[10,11], \Sigma_{0}$ can be written in terms of the Green function $G\left(r, r^{\prime}, \omega\right)$ and the retarded screened interaction $W\left(r, r^{\prime}, \omega\right)$. It has been shown that for STEM energy loss problems, a local dielectric function provides a good description of the excitations of the target [12]. The screened interaction $W\left(r, r^{\prime}, \omega\right)$ is the solution of Poisson's equation for a unit charge at rest at $r^{\prime}$.

The averaged self-energy of the incoming electron is given by

$$
\Sigma_{0}=\sum_{f} \int_{0}^{\infty} \frac{\mathrm{d} \omega}{\pi} \int \mathrm{d} \boldsymbol{r} \mathrm{d} r^{\prime} \frac{\left[\Phi_{0}^{*}(r) \Phi_{0}\left(r^{\prime}\right) \Phi_{\mathrm{f}}^{*}\left(r^{\prime}\right) \Phi_{\mathrm{f}}(r)\right]}{\omega-E_{0}+E_{\mathrm{f}}-\mathrm{i} \eta} \operatorname{Im}\left[W\left(\boldsymbol{r}, \boldsymbol{r}^{\prime}, \omega\right)\right]
$$

where $\Phi_{0}, \Phi_{\mathrm{f}}$ are the initial and final wave functions of the probe and $E_{0}$ and $E_{\mathrm{f}}$ the corresponding values of the energy [13]. $\eta$ is a positive infinitesimal.

We now apply the above expression to the case of a well focused STEM beam parallel to the $x$ axis, crossing at impact parameter $b$ from the target. In this case we obtain

$$
P(\omega)=\frac{1}{\pi v^{2}} \int \mathrm{d} x \mathrm{~d} x^{\prime} \operatorname{Im}\left\{W\left(r, r^{\prime}, \omega\right) \exp \left[\mathrm{i} \frac{\omega}{v}\left(x^{\prime}-x\right)\right]\right\}
$$

where the function $W\left(\boldsymbol{r}, \boldsymbol{r}^{\prime}, \omega\right)$ is evaluated at the trajectory $r=(b, x)$. This expression leads to the known formulae for simple targets such as films, cylinders or spheres, and is very useful to study more complicated geometries.

We apply this expression to compute the energy loss probability corresponding to a small particle coupled with an infinite planar surface. In this case the screened interaction in the region where the electron is moving can be written as follows

$$
\begin{aligned}
W\left(r, r^{\prime}, \omega\right)= & \frac{2}{\epsilon_{1}+\epsilon_{2}} \sum_{l=0}^{\infty} \sum_{m=-l}^{l} \eta_{l m} \frac{(l-m) !}{(l+m) !} \frac{r_{<}^{l}}{r_{>}^{l+1}} P_{l m}(\mu) P_{l m}\left(\mu^{\prime}\right) \exp \left[\mathrm{i} m\left(\phi-\phi^{\prime}\right)\right] \\
& +\sum_{l=0} \sum_{m=-l}^{l} A_{l m}\left(r^{\prime}, \mu^{\prime}\right) \frac{a^{l}}{r^{l+1}} P_{l m}(\mu) \exp \left[\mathrm{i} m\left(\phi-\phi^{\prime}\right)\right] .
\end{aligned}
$$

Here $\eta_{l m}=1\left(l+m\right.$ even) or $\epsilon_{2}(\omega) / \epsilon_{1}(\omega)(l+m$ odd $) ; r_{>}$and $r_{<}$are, respectively, the largest and the smallest of $r$ and $r^{\prime}$, and $P_{l m}$ are the Legendre functions. The first term 
of the sum in equation (3) corresponds to the combined effect of the Coulomb direct term and that induced by the image charge on the plane. This term leads to the well known expression for the stopping power of a particle moving close to a plane [14]. The second term in this expression gives the contribution of the small spherical particle to the induced field. The contribution of this term to the energy loss probability is

$$
\begin{gathered}
P(\omega)=\frac{2}{\pi v^{2}} \int_{0}^{\infty} \mathrm{d} x \int_{0}^{\infty} \mathrm{d} x^{\prime} \sum_{L} \sum_{m \geqslant 0}\left(2-\delta_{m 0}\right) \operatorname{Im}\left[A_{L m}\left(\boldsymbol{r}^{\prime}\right)\right] \frac{a^{L}}{r^{L+1}} P_{L m}(\mu)\left[\cos m\left(\phi(x)-\phi\left(x^{\prime}\right)\right)\right. \\
\left.\times \cos (\omega / v)\left(x-x^{\prime}\right)+\cos m\left(\phi(x)+\phi\left(x^{\prime}\right)\right) \cos (\omega / v)\left(x+x^{\prime}\right)\right]
\end{gathered}
$$

where $\delta_{m 0}$ is the Kronecker delta. The polar coordinates $\phi(x)$ and $\mu(x)$ are evaluated on the electron trajectory.

We have evaluated expression (4) for the case of an $\mathrm{Al}$ sphere half embedded in a planar interface. In figure 1 we show the energy loss spectrum when the support is $\mathrm{Al}$ and $\mathrm{AlF}_{3}$. Experimental values of $\epsilon(\omega)$ have been used [15].

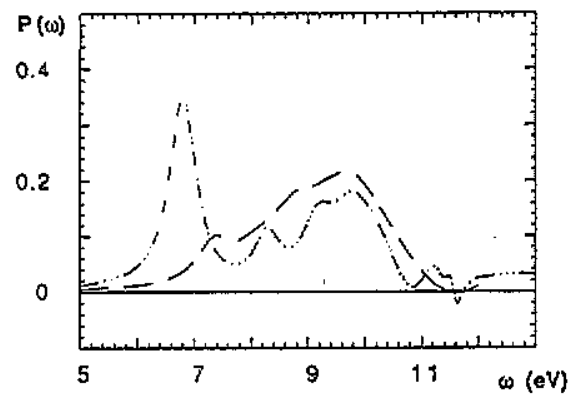

Figure 1. EEL spectra corresponding to a $10 \mathrm{~nm}$ Al sphere half-embedded in $\mathrm{Al}(-\cdots \rightarrow)$ and in $\mathrm{AlF}_{3}$ $(--)$. In both cases the beam crosses at grazing incidence close to the top of the sphere.

In the region of $8-10 \mathrm{eV}$ the calculated spectra are similar to the spectrum corresponding to an isolated sphere. The main effect of the metallic support is to give rise to a new resonance at $6.8 \mathrm{eV}$, which occurs neither for the insulator support nor for the isolated sphere. Batson [3] has reported experimental EEL spectra for Al spheres supported by another larger sphere, showing one new resonance which did not occur when the support was an insulator. The dependence of this effect on the nature of the support agrees with our results.

\section{References}

[1] Fujimoto F and Komaki K 1968 J. Phys. Soc. Japan 251679

[2] Batson P E 1980 Solid State Commun. 34477

[3] Batson P E 1982 Phys. Rev. Lett. 49936

[4] Batson P E 1982 Ultramicroscopy 9277

[5] Schmeits M 1981 J. Phys. C: Solid State.Phys. 141203

[6] Kohl H 1983 Ultramicroscopy $11 \mathrm{S3}$

[7] Wang Z L and Cowley J M 1987 Uttramicroscopy 2177

[8] Ugarte D, Colliex C and Trebbia P 1992 Phys. Rev. B 454332

[9] Ferrell T L and Echenique P M 1985 Phys. Rev. Lett. 551526

[10] Hedin L and Lundqvist S 1969 Solid State Physics vol 23 (New York: Academic) p 1

[11] Manson J R and Ritchie R H 1981 Phys. Rev. B 244867

[12] Howie A and Milne R H 1984 J. Microsc. 136 279; 1985 Ultramicroscopy 18427

[13] Echenique P M, Bausells J and Rivacoba A 1987 Phys. Rev. B 351521

[14] Echenique P M and Pendry J B 1975 J. Phys. C: Solid State Phys. 82936

[15] Hagemann H J, Gudat W and Kunz C 1974 Desy Report SR74/7 